ACS Synthetic Biology

Synthesis and Enzymology of 2'-Deoxy-7-deazaisoguanosine Triphosphate and its Complement. A Second Generation Pair in an Artificially Expanded

\title{
Genetic Information System
}

Nilesh B. Karalkar ${ }^{1}$, Nicole A. Leal ${ }^{1,2}$, Myong-Sang Kim² ${ }^{2}$ and Steven A. Benner ${ }^{1,2 *}$

${ }^{1}$ Foundation for Applied Molecular Evolution (FfAME), 13709 Progress Blvd, Box7, Alachua, Florida 32615

${ }^{2}$ Firebird Biomolecular Sciences LLC, 13709 Progress Boulevard, Box 17, Alachua, Florida 32615

*To whom correspondence should be addressed. Tel: +1 386418 0347; Fax: +1 386418 8856;

E-mail: manuscripts@ffame.org 


\section{Experimental Section}

Materials. All chemicals were purchased from Sigma-Aldrich (Sigma-Aldrich, USA). Solvents were of laboratory grade.

Procedures (Organic Synthesis). General. Thin-layer chromatography (TLC) was performed on TLC glass sheets covered with silica gel 60 F254 (E-Merck, Catalog No M5715-7). Column flash chromatography (FC): silica gel 60 (Sigma-Aldrich). UV spectra were recorded on a Beckman DU-640 spectrophotometer. NMR spectra were measured on a Varian-300 spectrometers. Chemical shifts $(\delta)$ are in ppm relative to internal Me4Si or external $\mathrm{H}_{3} \mathrm{PO} 4$ $\left({ }^{31} \mathrm{P}\right)$. The $J$ values are given in hertz.

\section{Synthesis of Protected 7-deazaisoguanosine}
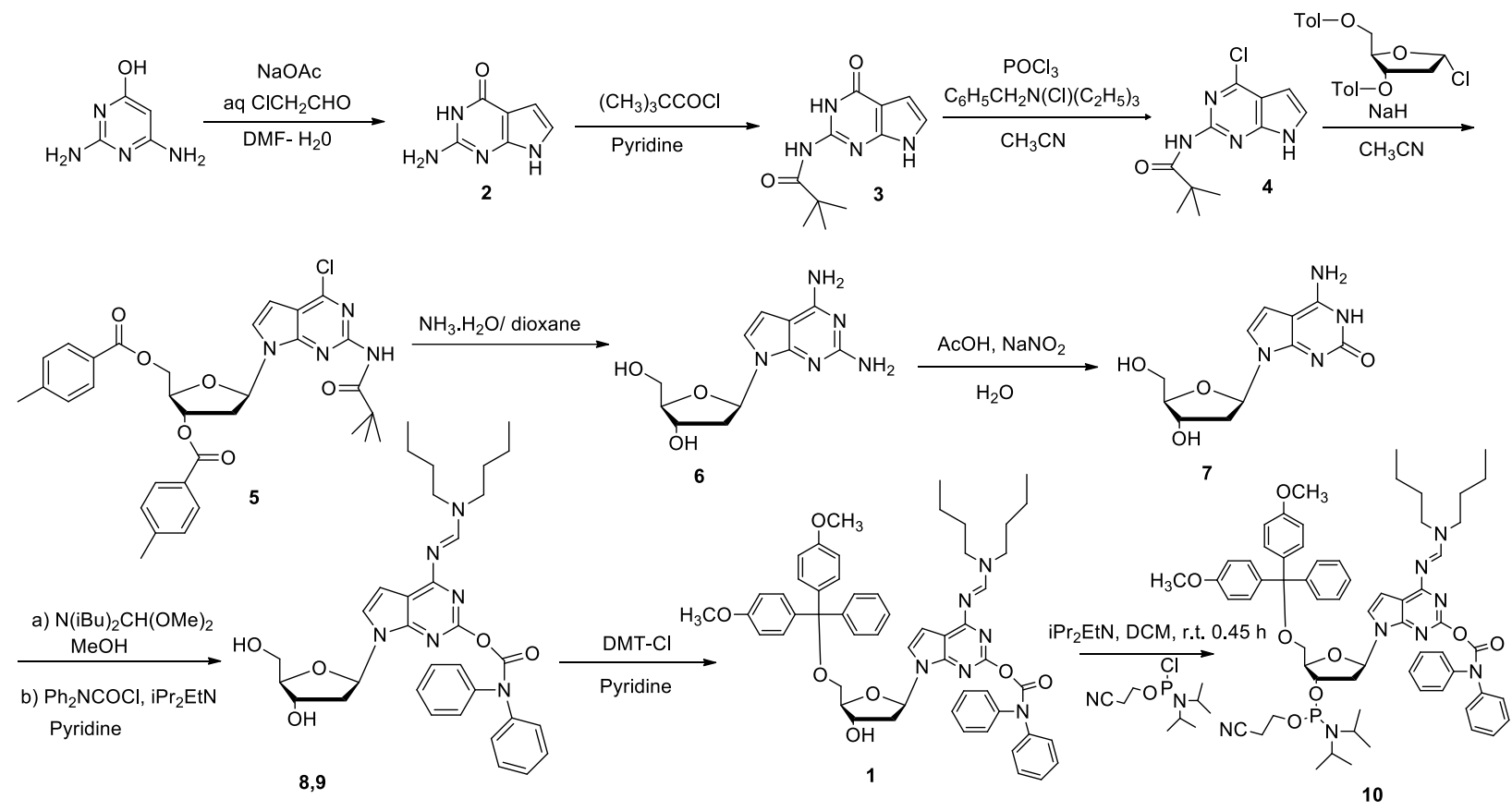

\section{2-Amino-3,7-dihydro-pyrrolo[2,3-d]pyrimidin-4-one (2).}

2,4-Diamino-6-hydroxypyrimidine (25.2 g, $200 \mathrm{mmol}$ ) was dissolved in DMF (480 mL) and water $(80 \mathrm{~mL})$ at room temperature. Sodium acetate $(16.6 \mathrm{~g}, 200 \mathrm{mmol})$ was added to this solution and the resulting yellow solution was stirred for $1 \mathrm{~h}$. Chloroacetaldehyde ( $25.3 \mathrm{~mL}, 200$ $\mathrm{mmol}$ ) was added, and the mixture was stirred for $46 \mathrm{~h}$ at room temperature. The reaction 
mixture was then concentrated by rotary evaporation. The product was triturated with water (20 $\mathrm{mL})$ and recovered by filtration. The filtered solid was digested in refluxing methanol $(500 \mathrm{~mL})$ for $2 \mathrm{~h}$, and the mixture was then placed in a refrigerator at $4{ }^{\circ} \mathrm{C}$ overnight to yield a product as a precipitate, which was recovered by filtration, washed with EtOAc $(4 \times 250 \mathrm{~mL})$ and dried in a vacuum desiccator over $\mathrm{P}_{2} \mathrm{O}_{5}$ (20 g, $133 \mathrm{mmol}, 66 \%$ yield).

${ }^{1} \mathrm{H}$ NMR (300 MHz, DMSO-d6) ppm 11 (s, 1 H), 10.35 (s, 1 H), 6.6 (s, 1 H), 6.15 (s, 1 H), 6.09 $(\mathrm{s}, 2 \mathrm{H})$

\section{N-(4-oxo-4,7-dihydro-3H-pyrrolo[2,3-d]pyrimidin-2-yl)pivalamide (3).}

A solution of 2-amino-3,7-dihydro-pyrrolo[2,3-d]pyrimidin-4-one (25 g, $166.66 \mathrm{mmol})$ in pyridine $(300 \mathrm{~mL})$ was treated with trimethylacetyl chloride $(65.74 \mathrm{~mL}, 533 \mathrm{mmol})$ at $90^{\circ} \mathrm{C}$ for $2 \mathrm{~h}$, to give a mixture of $\mathrm{N}(2)$-monoacylated and $\mathrm{N}(2), \mathrm{N}(7)$-bisacylated material. The solvent was evaporated and the residue was taken up in aqueous ammonia $\left(28 \% \mathrm{NH}_{3}, 42 \mathrm{~mL}\right)$ and $\mathrm{MeOH}(300 \mathrm{~mL})$, and stirred at room temperature for $30 \mathrm{~min}$, to selectively cleave the $\mathrm{N}(7)$ pivaloyl group. The product precipitates, and the solid was collected by filtration, washed with cold $\mathrm{MeOH}$, and dried on high vacuum (16 g, $68 \mathrm{mmol}, 41 \%$ yield).

${ }^{1} \mathrm{H}$ NMR (300 MHz, DMSO-d6) ppm 11.82 (s, $1 \mathrm{H}$ ), 11.58 (s, 1H), 10.8 (s., $\left.1 \mathrm{H}\right), 6.9$ (d, J=3.4 $\mathrm{Hz}, 1 \mathrm{H}), 6.38$ (d, J=3.6 Hz, $1 \mathrm{H}), 1.2(\mathrm{~s}, 9 \mathrm{H})$

\section{N-(4-chloro-7H-pyrrolo[2,3-d]pyrimidin-2-yl)pivalamide (4).}

A mixture of N-(4-oxo-4,7-dihydro-3H-pyrrolo[2,3-d]pyrimidin-2-yl) pivalamide (11 g, 47 mmol), $\mathrm{POCl}_{3}(26 \mathrm{~mL}, 282 \mathrm{mmol})$, benzyltriethylammonium chloride $(21.4 \mathrm{~g}, 94 \mathrm{mmol}), \mathrm{N}, \mathrm{N}-$ dimethylaniline (12 mL, $94 \mathrm{mmol})$, and acetonitrile $(104 \mathrm{~mL})$ was heated at reflux for $1 \mathrm{~h}$. The volatiles were removed by rotary evaporation, and the residual oil was slowly added to $800 \mathrm{~mL}$ of ice-water (which destroys the remaining $\mathrm{POCl}_{3}$ ). The $\mathrm{pH}$ was adjusted to 4 by dropwise addition of $28 \%$ aqueous $\mathrm{NH}_{4} \mathrm{OH}$ to generate product as a precipitate, which was collected by filtration, washed with cold water, and purified by silica chromatography (30\% ethyl acetate/hexane) to give purified product as a white solid ( $7 \mathrm{~g}, 0.27 \mathrm{~mol}, 58 \%$ yield).

${ }^{1} \mathrm{H}$ NMR (300 MHz, DMSO-d6) ppm 12.33 (br. s., $1 \mathrm{H}$ ), 10.04 (s, $\left.1 \mathrm{H}\right), 7.52$ (d, J=3.57 Hz, 1 H), $6.50(\mathrm{~d}, J=3.43 \mathrm{~Hz}, 1 \mathrm{H}), 1.20(\mathrm{~s}, 9 \mathrm{H})$ 
7-deaza-[2-deoxy-3,5-di-O-(p-toluoyl)- $\beta$-D-erythropentofuranosyl]-2-pivaloylamino-7Hpyrrolo[2,3-d]pyrimidine (5).

To a suspension of $\mathrm{NaH}(60 \%$ emulsion in oil, $1.1 \mathrm{~g}, 24 \mathrm{mmol})$ in dry acetonitrile $(400 \mathrm{~mL})$ was added $\mathrm{N}$-(4-chloro-7 $\mathrm{H}$-pyrrolo[2,3-d]pyrimidin-2-yl)-2,2-dimethyl-propionamide (6.0 g, 24 $\mathrm{mmol}$ ) at room temperature. After incubation for $1 \mathrm{~h}, 2$-deoxy-3, 5-di-O-( $p$-toluoyl)- $\beta$-D-erythropentofuranosyl chloride $(9.8 \mathrm{~g}, 25.2 \mathrm{mmol})$ was added to the reaction mixture, which was stirred further for $16 \mathrm{~h}$. The product (10 g, $16.52 \mathrm{mmol}, 68 \%$ yield) was obtained as a white solid after removal of the solvent on a rotary evaporator and purification by silica gel chromatography (EtOAc/hexanes 1:4).

${ }^{1} \mathrm{H} \mathrm{NMR}\left(300 \mathrm{MHz}, \mathrm{CDCl}_{3}\right) \operatorname{ppm} 8.15(\mathrm{~s}, 1 \mathrm{H}), 7.98(\mathrm{~d}, J=8.1 \mathrm{~Hz}, 2 \mathrm{H}), 7.90(\mathrm{~d}, J=8.1 \mathrm{~Hz}, 2$ H), 7.33 - $7.17(\mathrm{~m}, 6 \mathrm{H}), 6.75(\mathrm{t}, J=7.1 \mathrm{~Hz}, 1 \mathrm{H}), 6.51(\mathrm{~d}, J=3.8 \mathrm{~Hz}, 1 \mathrm{H}), 5.83-5.74(\mathrm{~m}, 1 \mathrm{H})$, 4.79 - 4.67 (m, $1 \mathrm{H}), 4.67$ - 4.52 (m, $2 \mathrm{H}), 3.12$ - 2.94 (m, $1 \mathrm{H}), 2.78$ (ddd, $J=2.3,6.0,14.3 \mathrm{~Hz}$, $1 \mathrm{H}), 2.42(\mathrm{~d}, J=7.7 \mathrm{~Hz}, 6 \mathrm{H}), 1.34(\mathrm{~s}, 9 \mathrm{H})$

7-deaza-(2-deoxy- $\beta$-D-erythro-pentofuranosyl)-7H-pyrrolo[2,3-d]pyrimidine-2,4-diamine (6). A suspension of 7-deaza-[2-deoxy-3,5-di-O-(p-toluoyl)- $\boldsymbol{\beta}$-D-erythropentofuranosyl]-2pivaloylamino-7 $H$-pyrrolo[2,3- $d$ ]pyrimidine $(4.96 \mathrm{~g}, 8.4 \mathrm{mmol})$ in dioxane $(80 \mathrm{~mL})$ and aqueous $28 \% \mathrm{NH}_{4} \mathrm{OH}(240 \mathrm{~mL})$ was transferred into an Parr bomb and stirred at $120{ }^{\circ} \mathrm{C}$ for $24 \mathrm{~h}$. The clear solution was concentrated by rotary evaporation, and the product was purified by flash chromatography on silica gel (5\% MeOH in DCM to 9:1 DCM: MeOH to 8:1.7:0.3 EtOAc: MeOH: $\left.\mathrm{H}_{2} \mathrm{O}\right)(1.66 \mathrm{~g}, 6.2 \mathrm{mmol}, 75 \%$ yield).

${ }^{1} \mathrm{H}$ NMR (300 MHz, DMSO-d6) ppm 7.40 (br. s., $2 \mathrm{H}$ ), 7.01 (d, $J=3.6 \mathrm{~Hz}, 1 \mathrm{H}$ ), 6.47 (d, $J=3.6$ Hz, 1 H), 6.22 - 6.38 (m, 3 H), 5.22 (d, J=2.9 Hz, 1 H), 4.27 (br. s., 1 H), 3.75 (br. s., 1 H), 3.40 $3.56(\mathrm{~m}, 2 \mathrm{H}), 2.27-2.42(\mathrm{~m}, 1 \mathrm{H}), 2.05(\mathrm{dd}, J=12.8,5.7 \mathrm{~Hz}, 1 \mathrm{H})$

\section{4-Amino-3-hydro-7-(2-deoxy- $\beta$-D-ribofuranosyl)-pyrrolo [2,3-d]-2-one (7)}

To a stirred solution of 7-deaza-(2-deoxy- $\boldsymbol{\beta}$-D-erythro-pentofuranosyl)-7H-pyrrolo-[2,3-d]pyrimidine-2,4-diamine (5 g, $19 \mathrm{mmol})$ in $300 \mathrm{~mL}$ of $20 \% \mathrm{AcOH}-\mathrm{H}_{2} \mathrm{O}(\mathrm{v} / \mathrm{v})$, was added dropwise a solution of $\mathrm{NaNO}_{2}(2.75 \mathrm{~g}, 39.9 \mathrm{mmol})$ in water $(57 \mathrm{~mL})$ at room temperature. The stirring was continued for $110 \mathrm{~min}$, after which the $\mathrm{pH}$ of the dark solution was adjusted to 6.0 
with $28 \%$ aqueous $\mathrm{NH}_{3}$. The solution was applied to a Amberlite XAD-16N column (resin 20-60 mesh), the column was washed with $\mathrm{H}_{2} \mathrm{O}(500 \mathrm{~mL})$, and the product was eluted with $\mathrm{H}_{2} \mathrm{O}-i$ $\mathrm{PrOH}$ (80:20). After rotary evaporation, the product was obtained as dark brown solid (3.34 g, 12.55 mmol, $66 \%$ yield).

${ }^{1}$ H NMR (300 MHz, DMSO-d6) ppm 7.92 (br. s., 2 H), 6.89 (d, J=3.7 Hz, 1 H), 6.39 (d, J=3.6 Hz, 1 H), 6.19 (dd, J=7.8, 6.2 Hz, 1 H), 4.22 - 4.30 (m, 1 H), 3.71 - 3.79 (m, 1 H), 3.42 - 3.55 (m, $2 \mathrm{H}), 2.26-2.39(\mathrm{~m}, 1 \mathrm{H}), 1.98-2.09(\mathrm{~m}, 1 \mathrm{H})$

\section{1-(2-Deoxy - $\beta$-D-erythro-pentofuranosyl)-4-( $N, N$-dibutylaminomethylidene $)-6$ -}

[(diphenylcarbamoyl)oxy]-1H-pyrrolo[2,3-d] pyrimidine (9).

To a stirred suspension of 7 (4 g, $15 \mathrm{mmol})$ in methanol $(25 \mathrm{~mL})$ was added N, N-dibutylformamide dimethyl acetal $(7.3 \mathrm{~mL}, 31.62 \mathrm{mmol})$. The mixture was stirred at room temperature overnight and evaporated to dryness by rotary evaporation. The product was obtained as a brown foam of $\mathbf{8}(3.8 \mathrm{~g}, 9.4 \mathrm{mmol}, 63 \%$ yield) following purification by flash chromatography (silica gel, $\mathrm{CH}_{2} \mathrm{Cl}_{2}-\mathrm{MeOH}$ 9:1 to 8.5: 1.5) using a stepwise gradient.

${ }^{1} \mathrm{H}$ NMR (300 MHz, DMSO-d6) ppm 11.20 (br. s., $\left.1 \mathrm{H}\right), 8.41$ (s, $\left.1 \mathrm{H}\right), 7.07$ (d, J=3.8 Hz, $1 \mathrm{H}$ ), 6.19 - 6.35 (m, 2 H), 5.25 (br. s., 2 H), 4.30 (br. s., 1 H), 3.79 (d, J=2.1 Hz, 1 H), 3.30 - 3.60 (m, $7 \mathrm{H}), 2.28$ - 2.45 (m, $1 \mathrm{H}), 1.99$ - 2.15 (m, $1 \mathrm{H}), 1.58$ (quin, J=7.3 Hz, $4 \mathrm{H}), 1.18$ - 1.40 (m, 4 H), $0.82-1.01(\mathrm{~m}, 6 \mathrm{H})$

${ }^{13} \mathrm{C}$ NMR (75 MHz, DMSO-d6) ppm 158.2, 157.5, 156.8, 122.3, 101.6, 99.6, 87.8, 83.4, 71.8, 62.8, 51.6, 45.1, 41.0, 40.7, 40.4, 40.1, 39.9, 39.8, 39.6, 39.3, 30.9, 29.1, 20.2, 19.8, 14.3, 14.2 To a solution of $\mathbf{8}(3.8 \mathrm{~g}, 9.4 \mathrm{mmol})$ in dry pyridine $(20 \mathrm{~mL})$ were added diphenylcarbamoyl chloride ( $3.92 \mathrm{~g}, 17 \mathrm{mmol}$ ) and N,N-diisopropylethylamine (2.94 mL, $17 \mathrm{mmol})$. The mixture was stirred for $1 \mathrm{~h}$ at room temperature, and then poured in the $5 \%$ aqueous $\mathrm{NaHCO}_{3}(250 \mathrm{~mL})$ and extracted with $\mathrm{CH}_{2} \mathrm{Cl}_{2}(2 \times 250 \mathrm{~mL})$. The combined $\mathrm{CH}_{2} \mathrm{Cl}_{2}$ layers were dried over $\mathrm{Na}_{2} \mathrm{SO}_{4}$ and clarified by filtration. The product 9 was then recovered by rotary evaporation and purified by flash chromatography (silica gel, elution with $\mathrm{CH}_{2} \mathrm{Cl}_{2}$ followed by $\mathrm{CH}_{2} \mathrm{Cl}_{2}-\mathrm{MeOH}$ step wise from 0 to $4 \%$ methanol) to give a brown color foam ( $5 \mathrm{~g}, 8.3 \mathrm{mmol}, 89 \%$ yield).

${ }^{1} \mathrm{H}$ NMR (300 MHz, DMSO-d6) ppm 8.71 (s, $\left.1 \mathrm{H}\right), 7.37$ - 7.50 (m, $\left.10 \mathrm{H}\right), 7.24$ - 7.34 (m, $\left.2 \mathrm{H}\right)$, 6.52 - 6.37 (m, 2 H), 5.30 (d, J=4.0 Hz, 1 H), 4.98 (t, J=5.6 Hz, 1 H), 4.28 - 4.37 (m, 1 H), 3.77 - 
$3.84(\mathrm{~m}, 1 \mathrm{H}), 3.38$ - $3.61(\mathrm{~m}, 6 \mathrm{H}), 2.09$ - $2.21(\mathrm{~m}, 1 \mathrm{H}), 1.48$ - $1.65(\mathrm{~m}, 4 \mathrm{H}), 1.18$ - $1.38(\mathrm{~m}, 4$ $\mathrm{H}), 0.90(\mathrm{q}, J=7.3 \mathrm{~Hz}, 6 \mathrm{H})$

${ }^{13} \mathrm{C}$ NMR (75 MHz, DMSO-d6): ppm 162.6, 157.5, 156.0, 152.8, 152.5, 142.6, 129.9, 127.8, 127.5, 124.5, 110.0, 101.3, 88.0, 83.5, 71.8, 62.7, 51.6, 45.1, 40.9, 40.7, 40.4, 40.1, 39.8, 39.6, 39.3, 31.1, 29.2, 20.2, 19.8, 14.3, 14.2.

\section{1-[2-Deoxy-5-O-(4,4-dimthoxytrityl)- $\beta$-D-erythropentofuranosyl]-4- $(N, N-$} dibutylaminomethylidene)-6-[(diphenylcarbamoyl)-oxy]-1H-pyrrolo[2,3-d]pyrimidine (1) Compound 9 (5 g, $8.3 \mathrm{mmol})$ was dried by co-evaporation with anhydrous pyridine $(2 \mathrm{x}, 15 \mathrm{~mL})$ and dissolved in anhydrous pyridine $(100 \mathrm{~mL})$. The solution was treated with dimethoxytrityl chloride (3.1 g, $9.1 \mathrm{mmol}$ ) at room temperature under stirring for $4 \mathrm{~h}$. Water was then added to the mixture and the stirring was continued for $35 \mathrm{~min}$. The mixture was diluted with a 5\% aqueous $\mathrm{NaHCO}_{3}$ solution $(100 \mathrm{~mL})$ and extracted with $\mathrm{CH}_{2} \mathrm{Cl}_{2}(2 \times 350 \mathrm{~mL})$. The combined extracts were dried over $\mathrm{Na}_{2} \mathrm{SO}_{4}$. The solvent was removed by rotary evaporation, and the product 1 was obtained as an orange-white foam $(5 \mathrm{~g}, 5.55 \mathrm{mmol}, 66 \%$ yield $)$ by purification by flash chromatography (silica gel, eluted with 2:1 hexane:ethyl acetate).

${ }^{1} \mathrm{H}$ NMR (300 MHz, $\left.\mathrm{CDCl}_{3}\right)$ : ppm 8.77 (s, $\left.1 \mathrm{H}\right), 7.07$ - 7.45 (m, $\left.22 \mathrm{H}\right), 6.80$ (d, J=8.8 Hz, $\left.4 \mathrm{H}\right)$, $6.71(\mathrm{t}, J=6.7 \mathrm{~Hz}, 1 \mathrm{H}), 6.55(\mathrm{~d}, J=3.7 \mathrm{~Hz}, 1 \mathrm{H}), 4.49$ - $4.57(\mathrm{~m}, 1 \mathrm{H}), 4.01$ (q, J=4.1 Hz, $1 \mathrm{H})$, 3.78 (s, $6 \mathrm{H}), 3.56$ - 3.64 (m, 2 H), 3.29 - 3.38 (m, 4 H), 2.33 - 2.53 (m, 2 H), 2.28 (d, J=3.4 Hz, $1 \mathrm{H}), 1.54$ - 1.73 (m, $4 \mathrm{H}), 1.22$ - $1.44(\mathrm{~m}, 4 \mathrm{H}), 0.95$ (td, J=7.3, 2.3 Hz, $6 \mathrm{H})$

${ }^{13} \mathrm{C}$ NMR (75 MHz, $\mathrm{CDCl}_{3}$ ): ppm 162.6, 158.7, 157.2, 156.3, 153.2, 152.9, 144.9, 142.6, 136.0, 130.3, 129.1, 128.4, 128.1, 126.4, 122.2, 113.3, 110.3, 101.8, 86.6, 85.0, 82.8, 77.6, 77.2, 76.8, 73.0, 64.3, 55.4, 52.0, 45.3, 40.9, 31.3, 29.2, 20.3,14.01.

\section{1-[2-Deoxy-5-O-(4,4'-dimthoxytriphenylmethyl)- $\beta$-D-erythro-pentofuranosyl]-4- $(N, N-$} dibutylaminomethylidene)-6-[(diphenylcarbamoyl) oxy-1-H-pyrralo[2,3-d] pyrimidine 3'-(2cyanoethyl-N,N-diisopropyl) phosphoramidite. (10).

A solution of compound $1(0.2 \mathrm{~g}, 0.22 \mathrm{mmol})$ in $\mathrm{CH}_{2} \mathrm{Cl}_{2}(10 \mathrm{~mL})$ was pre flushed with argon and kept under argon atmosphere. 2-Cyanoethyldiisopropylphosphoramidochloridite $(0.156 \mathrm{~mL}$, $0.704 \mathrm{mmol})$ and $\mathrm{N}, \mathrm{N}$-diisopropylethylamine $(0.155 \mathrm{~mL}, 0.89 \mathrm{mmol})$ were added to above solution at room temperature. Stirring was continued for $45 \mathrm{~min}$, at which point the mixture was added to $50 \mathrm{~mL}$ of an aqueous solution of $5 \% \mathrm{NaHCO}_{3}$. The mixture was shaken, the layers were 
separated, and the aqueous layer was extracted with $\mathrm{CH}_{2} \mathrm{Cl}_{2}(3 \times 50 \mathrm{~mL})$. The combined organic extracts were dried over $\mathrm{Na}_{2} \mathrm{SO}_{4}$, filtered, rotary evaporated, and applied to flash

chromatography (silica 60, spherically, neutral, eluted with $\mathrm{CH}_{2} \mathrm{Cl}_{2}$-acetone (9:1)) to give product 11 as a white foam $(0.153 \mathrm{~g}, 0.138 \mathrm{mmol}, 63 \%$ yield $)$.

${ }^{1} \mathrm{H}$ NMR (300 MHz, $\mathrm{CdCl}_{3}$ ) ppm 8.77 (s, $\left.1 \mathrm{H}\right), 7.14$ - 7.47 (m, $\left.20 \mathrm{H}\right), 6.67$ - 6.84 (m, $\left.5 \mathrm{H}\right), 6.56$

$(\mathrm{d}, J=2.6 \mathrm{~Hz}, 1 \mathrm{H}), 4.62$ - $4.74(\mathrm{~m}, 1 \mathrm{H}), 4.12$ - 4.19 (m, $1 \mathrm{H}), 3.76$ (d, J=1.8 Hz, $6 \mathrm{H}), 3.52$ - 3.67

(m, 4 H), 3.29 - 3.44 (m, 3 H), 3.25 (br. s., 1 H), 2.38 - 2.57 (m, 13 H), 1.62 (m, 4 H), 1.29 - 1.44

(m, $4 \mathrm{H}), 1.15$ (m, $8 \mathrm{H}), 0.91$ - 1.08 ppm (m, $23 \mathrm{H})$

${ }^{31} \mathrm{P}$ NMR (121 $\left.\mathrm{MHz} \mathrm{CDCl}_{3}\right): \mathrm{ppm}=148.5,148.4$.

$[\mathrm{M}+\mathrm{H}]^{+}=1103.5518$

$[\mathrm{M}+\mathrm{Na}]^{+}=1125.5338$ 